\title{
Evaluación estructural y funcional de los sistemas productivos urbanos en la ciudad de Quibdó, Chocó, Colombia
}

\section{Structural and functional evaluation of the urban productive systems in the city of Quibdo, Choco, Colombia}

\author{
Teófilo Cuesta-Borja ${ }^{1}$; Ditter Horacio Mosquera ${ }^{2}$ \\ RESUMEN
}

La mayor parte de la agricultura urbana permanece todavía en gran medida inexplorada y sin recibir reconocimiento y apoyo por parte de las entidades gubernamentales. Con el objetivo de caracterizar de manera estructural y funcional los sistemas productivos urbanos en Quibdó, Colombia, se analizó la vegetación por hábito de crecimiento y se hizo un diagnóstico rural rápido (DRR) sobre la utilización de las especies. El diagnóstico incluyó nivel de utilización de especies, importancia biofísica y demanda comercial. Los resultados indican que los habitantes de Quibdó utilizan como espacios para desarrollar la agricultura urbana el huerto casero mixto, la azotea, cultivo en materas y cría de animales. Se encontraron 58 especies incluidas en 51 géneros y 41 familias botánicas, distribuidas en el huerto en tres estratos: arbóreo, arbustivo y herbáceo. En el estrato arbóreo, Inga edulis, Bactris gasipaes y Zisigyum malacensis presentaron mayor CIE; en arbustivo, las representativas fueron, Musa spp, Carica papaya y Citrus lemon; en herbáceo, Ocimum $s p$, Eringyum foetidum y Petiveria alliacea. La importancia de estas especies, radica en su utilización en alimentación y medicina tradicional. Como conclusión se tiene que los sistemas productivos urbanos en Quibdó se orientan a suplir necesidades alimentarias y medicinales.

Palabras clave: Agricultura urbana; Caracterización; Coeficiente de importancia de la especie; Diagnóstico rural rápido; Huerto casero mixto; Azotea.

\section{ABSTRACT}

Most of urban agriculture remains still to a great extent unexplored and without receiving recognition and support on the part of the governmental organizations. With the objective of characterizing structurally and functionally the urban productive systems in Quibdo, Colombia, the vegetation were analyzed by growth habit and a fast rural diagnosis became (DRR) on the use of the species. The diagnosis included level of use of species, biophysic importance and commercial demand. The results indicate that the inhabitants of Quibdo use like spaces to develop to urban agriculture the mixed homemade orchard, the roof and culture in materas, and animal. Were 58 species including in 51 Sorts and 41 botanical families, distributed in the orchard in three layers: arboreal, bush, herbaceous. In the arboreal layer, Inga edulis, Bactris gasipaes and Zisigyum malacensis presented major CIE; in bush, the representative ones were, Musa spp, Carica papaya and Citrus lemon; in herbaceous, Ocimum $s p$, Eringyum foetidum and Alliacea petiveria. The importance of these species is in its use in feeding and traditional medicine.
1. Ingeniero Agrónomo, Especialista en Gestión Ambiental. Subdirector Científico, Instituto de Investigaciones Ambientales del Pacífico (IIAP), Presidente Fundación Agricultura con Enfoque Humano (FAEH). e-mail:tcuesta@iiap.org.co, tcuesta@earth.ac.cr

2. Ingeniero Agroforestal, Estudiante de Maestría en Agroforestería Tropical, Investigador Universidad Tecnológica del Chocó, Quibdó, Colombia.

e-mail:dmosquera@catie.ac.cr

Recibido: 20 de enero de 2010

Aceptado: 3 de febrero de 2010 
As conclusion has them urban productive systems in Quibdó are oriented to replace nourishing and medicinal needs.

Keywords: Urban agriculture; Characterization; Coefficient of importance of the species; Fast rural diagnosis; Mixed homemade; Azotea.

\section{INTRODUCCCIÓN}

La Organización de las Naciones Unidas para la Agricultura y la Alimentación $(\mathrm{FAO})$ señala que la agricultura en áreas urbanas y peri-urbanas proporciona ya alimentos a cerca de 700 millones de residentes en las ciudades, un cuarto de la población urbana mundial, y afirma que en la actualidad los pobres urbanos de muchos países en desarrollo gastan al menos $60 \%$ de sus ingresos en comida, lo que lleva a la conclusión de que las explotaciones agrícolas en el interior o la periferia de áreas urbanas jugarán un papel cada vez mayor para alimentar a su población. No obstante, las iniciativas de los «agricultores urbanos» de países en desarrollo se adelantan a menudo de manera informal y aislada, sin estar ligadas a programas gubernamentales (FAO 1999).

Por otra parte, a nivel mundial se estima que «La mayor parte de la agricultura urbana permanece todavía en gran medida inexplorada y sin recibir reconocimiento y apoyo por parte de las entidades gubernamentales» (Mougeot 1993). Caso similar ocurre en las ciudades de Colombia, a lo que la ciudad de Quibdó no escapa, porque a pesar de que se conocen los privilegios socio-ambientales y culturales de la implementación de la agricultura urbana, no se han documentado experiencias tendientes a caracterizar su dinámica estructural y funcional, teniendo en cuenta las prácticas utilizadas para su manejo, ni las especies que hacen parte de ésta, destacando las relaciones inter-específicas e intra-específicas con otras especies y el aporte que hacen a la economía, la belleza escénica o el sostenimiento de las tradiciones culturales de los pobladores del municipio de Quibdó.

Con base en lo anterior, este estudio se adelantó, con el objetivo de generar conocimiento básico de la estructura y composición de los sistemas de agricultura urbana desarrollados en la ciudad de Quibdó, lo que brindará información relevante que facilitará el proceso participativo de selección de especies dentro de acciones de mejoramiento de sistemas productivos, porque destaca la importancia ecológica, económica, socio-cultural y de uso que presentan las especies, lo que aporta elementos contundentes para juzgar su potencial capacidad para contribuir en la solución de problemas ambientales y socio-económicos que prevalecen en las zonas donde se establecen.

\section{ÁREADE ESTUDIO}

Este estudio se realizará en el municipio de Quibdó, ubicado a $5^{\circ} 40^{\prime} 30^{\prime \prime}$ latitud norte y $76^{\circ} 39^{\prime} 39^{\prime \prime}$ longitud este, a una altura de $54 \mathrm{msnm}$ en clima cálido húmedo, temperatura promedio mensual de $26.5^{\circ} \mathrm{C}$, precipitación media anual superior a $1000 \mathrm{~mm}$, humedad relativa de $86 \%$; corresponde este sitio a la zona de vida de bosque pluvial tropical (bp-T) (Rangel 2004)(Figura 1).

\section{MÉTODOS}

Diseño de instrumento de diagnóstico. Para la caracterización estructural y funcional de las especies del sistema, se eligieron en cada una de las comunas del municipio de Quibdó cinco sistemas de agricultura urbana típicos, tomando en consideración los distintos arreglos espaciales y la diversidad florística. Se diseñaron encuestas semiestructuradas para la recolección de la información sobre el uso, importancia de las especies y aspectos socio-económicos de los pobladores. Para la recolección de información sobre las especies de los sistemas de producción urbanos, se aplicaron formularios de campo elaborados para el inventario de recursos bióticos.

Levantamiento de información de campo. Para la recolección de datos de las especies, éstas se clasificaron en relación con su hábito ecológico, evaluado como altura total: arbórea $>5 \mathrm{~m}$; arbustivo $1 \mathrm{~m}$ a $5 \mathrm{~m}$ (incluyendo las lianas y trepadoras) y herbáceo $<1 \mathrm{~m}$. La estructura de los sistemas de producción se representó a través de perfiles estructurales idealizados de componentes bióticos (plantas animales) $(5 \times 15$ $\mathrm{m})$. Los datos socio-económicos y los datos del uso e importancia de las especies se obtuvieron a través de encuestas y observaciones directas, apoyadas por cuestionarios estructurados realizados a los pobladores (diagnóstico rural rápido) sobre el manejo y utilización de las especies cultivadas(Gama 1995).

Evaluación estructural y funcional. En la evaluación estructural y funcional de los sistemas se utilizó una ecuación que considera el nivel de utilización, la importancia biofísica y la demanda de las especies por los productores, denominada Coeficiente de Importancia de la Especie (CIE):

$$
\mathrm{CIE}=\frac{3 * \mathrm{NU}+2 * \mathrm{IB}+\mathrm{DC}}{6}
$$

donde:

3 y 2: unidades por las que se multiplica NU y IB según la importancia de la variable.

6: factor de ponderación de la ecuación.

Nivel de utilización (NU): expresa la importancia de la 


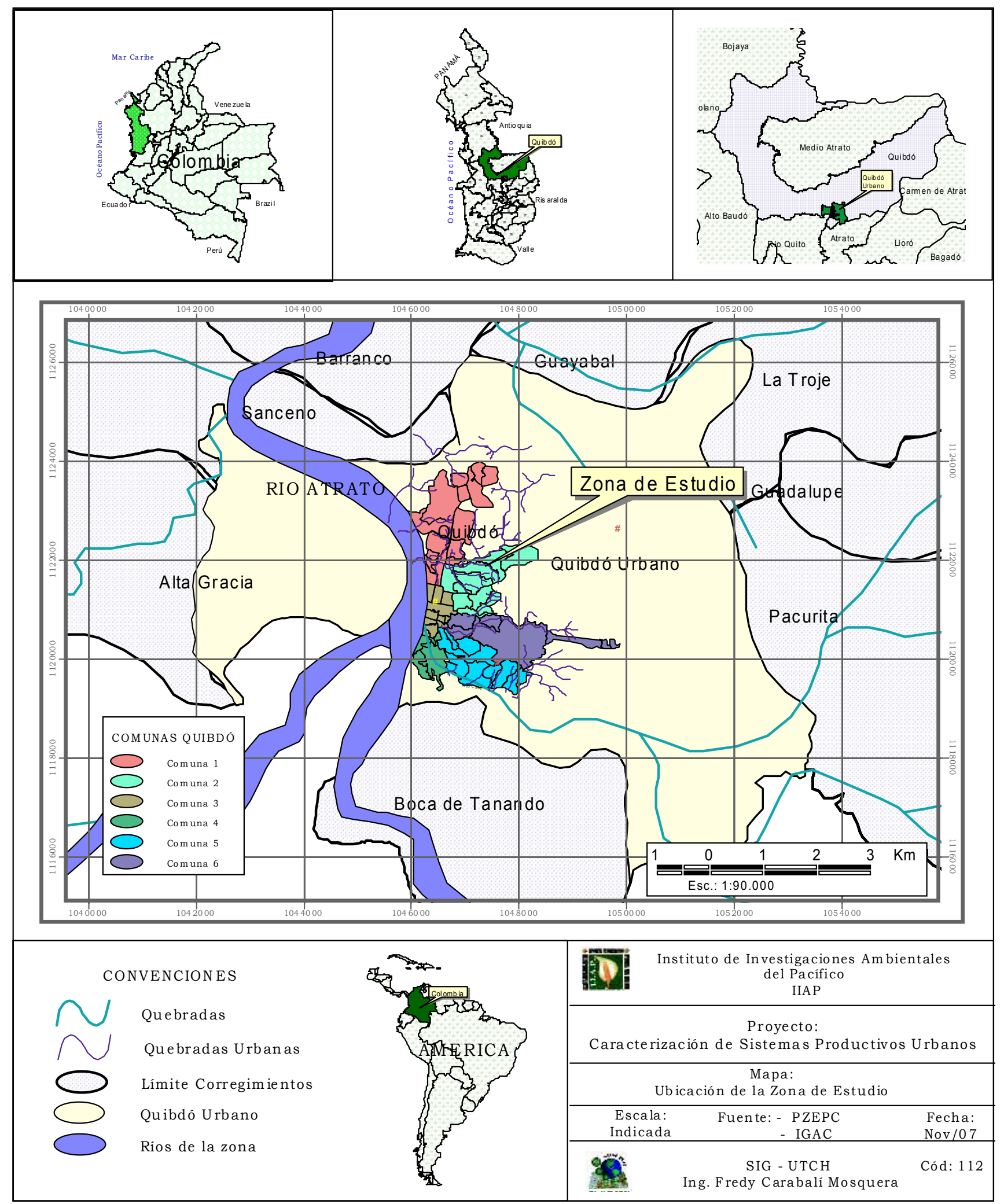

Figura 1. Ubicación geográfica de la zona de estudio. 
especie en cuanto a su funcionalidad para la familia:

3. Muy utilizada: especie con tres o más usos

2. Utilizada: especie con dos usos

1. Poco utilizada: especie con un uso

Importancia Biofísica(IB), representa la frecuencia de la especie:

3: alta - frecuencia $(70-100 \%)$

2: media - frecuencia $(31-69 \%)$

1: baja-frecuencia $(1-30 \%)$

0: muy baja - frecuencia $(0.1-0.9 \%)$

Demanda de comercialización (DC): expresa el potencial de comercialización de la especie:

3: alta - muy demandada

2: media - medianamentee demandada

1: baja - poco demandada

0 : inexistente

\section{RESULTADOSYDISCUSIÓN}

Principales sistemas de producción urbanos. Se encontraron básicamente tres tipos de sistemas de producción: el huerto casero mixto, la azotea y cultivo de plantas en utensilios domésticos y materas, y la cría de animales confinados.

El huerto casero mixto. Los agricultores urbanos los denominan «sembrados al lado de la casa»; en estos se siembran a manera de policultivo y alrededor de las viviendas, especies que reportan un uso alimenticio, medicinal o mágicoreligioso; entre estas especies se pueden citar como principales las denominadas «manchas» que incluyen el plátano y todas las especies del género Mussa (primitivo, cuatro filos, popochos, etc.), la bija, el chontaduro, el caimito, la guama, la guayaba, el almirajó, el bacao, guayaba dulce y agria, marañón, árbol del pan, chirimoya, guanábana, totumo, entre otras. Todas estas especies se establecen sin ningún tipo de arreglo. Es frecuente encontrar en los huertos caseros, la presencia de gallinas que se crían sin ningún tipo de confinamiento, en especial en barrios como Jardín, Esmeralda y Santa Ana, y con mayor frecuencia en barrios periféricos como Caraño, Futuro y Cascorba. Estos huertos caseros son manejados sobre todo por las mujeres y los jóvenes,

Los huertos caseros mixtos se han implementado por la mayoría de los pobladores chocoanos desde épocas inmemoriales y se constituyen en un componente fundamental de sus sistemas de producción agrícola. La variedad de cultivos que por lo común se establecen en un huerto casero, lo convierten en una despensa de múltiples productos (alimentos hortícolas, frutales, madera, leña, fibras, plantas medicinales y ornamentales), cuya producción es sostenible por un número indeterminado de años, por la similitud que poseen estos sistemas con el bosque natural, donde los efectos benéficos de la asociación de las diversas especies, promueve el ciclaje de nutrientes, que ayuda a mantener la fertilidad del suelo, favorece el control biológico de plagas y previene la erosión, entre otros beneficios. Lo anterior, sumado a la facilidad de manejo agronómico que requiere este sistema, lo convierten en una de las bases de la seguridad alimentaria y garantía de algunos ingresos económicos de muchas de las familias de las comunidades del Alto Atrato.

La azotea y cultivo de plantas en utensilios domésticos y materas. Es frecuente encontrar el cultivo de plantas medicinales (anamú, amansa justicia, albahaca del santísimo) y alimenticias (cilantro, albahaca, tomate, cebolla de rama, poleo, menta entre otras) en armazones comúnmente de denominadas «azoteas» construidas con productos de fácil consecución en la zona, como son las cantoneras (cortezas de árboles aserrados), tablas rústicas, guadua y en menor caso de cemento. Este sistema de producción agrícola, no deja de ser una huella del legado cultural de los pueblos de los cuales es oriunda la gente que hace su arribo por diferentes motivos a la ciudad de Quibdó. La presencia de azoteas tiene su mayor representatividad en barrios periféricos como Paraíso, Caraño, Fuego Verde, Cascorba, Futuro y Villa España.

En las zonas céntricas del municipio, se prefiere la siembra de un sinnúmero de plantas alimenticias y medicinales (albahaca, poleo, cilantro, etc.), y ornamentales y mágico-religiosas (amansa justicia, millonaria, anamú, etc.) en utensilios plásticos y de aluminio así como una gran variedad de materas comercialmente diseñadas para este fin.

Cría de animales confinados. Cada vez es más escaso encontrar en la ciudad de Quibdó sistemas de cría de animales confinados; esto se debe a que en algunas zonas las autoridades ambientales han extremado las medidas para regularla o prohibirla, por las molestias causadas a los vecinos por la emanación de malos olores; otra razón expuesta por algunos agricultores urbanos es la carencia de espacios para realizarla al interior de la ciudad. Otras personas (sobre todo en la zona norte) lo atribuyen a que no se posee un suministro de agua suficiente para realizar explotación pecuaria, sobre todo la porcina. Básicamente en la cría de animales confinados se utilizan las gallinas y rara vez cerdos u otros animales como patos y/o pavos. La mayor parte de la producción de la cría de animales confinados se destina para el autoconsumo de la familia, no queriendo decir esto que no se produzcan en ocasiones excedentes comercializables.

Análisis estructural. El área promedio de los sistemas productivos encontrados fue de $80 \mathrm{~m}^{2}$. Se identificaron 58 especies de importancia mágico-religiosa, medicinal, alimenticia y ornamental para la comunidad distribuidas en 51 géneros y 41 familias botánicas. De las especies encontradas $42 \%$ corresponden a plantas de hábito arbóreo, $22 \%$ a especies de hábito arbustivo y $36 \%$ a especies de hábito herbáceo. En el perfil idealizado de la Figura 2 se puede observar la distribución de las especies en los diferentes estratos, donde se encontró que Bactris gasipaes, Inga edullis, y Zisigyum 


\section{Bioetnia Volumen 7 № 1 (enero-junio), 2010}

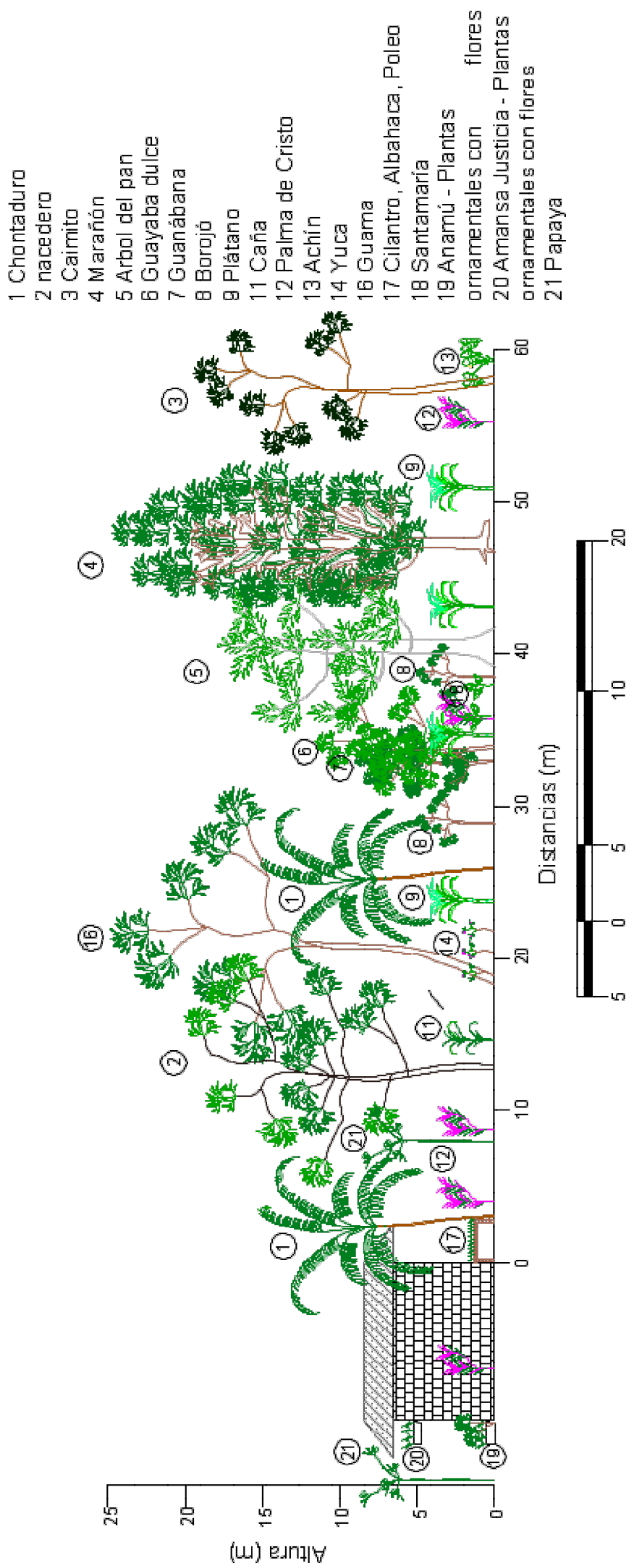

malacensis están entre las especies más representativas en el estrato arbóreo; Borojoa patinoi, Mussa sp, Cardyline terminalis, se destacan en el estrato arbustivo. Mientras que, Ocimum sp, Eringyum foetidum y Petiveria alliacea sobresalen en el estrato herbáceo.

El diagrama de frecuencia de la Figura 3, muestra que de las especies registradas en el estrato arbóreo, las que se encontraron con mayor frecuencia fueron Bactris gasipaes (75\%), Zisigyum malacensis (50\%), Inga edulis (75\%); en el estrato arbustivo, se destacan Mussa sp (75\%), Cardyline terminalis (75\%) y Anona muricata $(50 \%)$; entre las especies encontradas con mayor frecuencia en el estrato herbáceo se pueden citar Ocimum sp, Eringyum foetidum y Petiveria alliacea todas con una frecuencia de aparición de $75 \%$.

Usos de las especies. Las especies encontradas se asociaron a cuatro formas de uso: alimenticias, medicinal, mágico religioso, ornamental y otros usos. Considerando el uso por hábito arbóreo, se pudo determinar que entre las especies arbóreas, $80 \%$ son alimenticias, $28 \%$ son medicinales, $12 \%$ se usan para fines mágico-religiosos y $44 \%$ reportan otros usos alternativos; entre las arbustivas, $69 \%$ son alimenticias, $46 \%$ medicinales, $23 \%$ tienen usos mágico-religiosos y $38 \%$ poseen otros usos. De las especies herbáceas, $62 \%$ son alimenticias, $62 \%$ poseen usos medicinales, $19 \%$ usos mágico-religiosos, $33 \%$ poseen otros usos.

En las Tablas 1, 2 y 3, se anotan los usos de las especies que se encontraron según hábito ecológico.

Especies más importantes por hábito de crecimiento

Estrato arbóreo. Inga edulis es una especie que justifica su importancia al interior de los huertos caseros mixtos locales, porque se utiliza para múltiples propósitos; sus frutos (medianamente comercializables) son comestibles, sus hojas se usan con fines medicinales, su corteza se utiliza con fines mágicoreligiosos, sus troncos y ramas se emplean en la construcción de casas, cercas y como leña. Los frutos de Bactris gasipaes, muy comercializables, se utilizan para la alimentación humana y animal. Los frutos de Zisigium macensis además de presentar una alta demanda de comercialización para consumo directo, poseen una alta distribución al interior de la ciudad y es muy utilizado como árbol de sombra. El nivel de utilización, la importancia biofísica y la demanda de las especies de este hábito de crecimiento se muestra en la Tabla 4.

Estrato arbustivo. Musa spp cuenta con una alta demanda de comercialización y además es fundamental en la alimentación humana y animal local; se en- 
Sistemas productivos urbanos. T Cuesta-Borja, DH Mosquera

Tabla 1

Especies encontradas según hábito arbóreo

\begin{tabular}{|c|c|c|c|c|c|c|}
\hline \multirow{2}{*}{$\begin{array}{l}\text { Hábito ecológico } \\
\text { Arbórea }\end{array}$} & \multirow[t]{2}{*}{ Nombre local } & \multirow[t]{2}{*}{ Nombre científico } & \multicolumn{4}{|c|}{ Nivel de utilización } \\
\hline & & & Medicinal & Mágico religiosa & Alimento & Otros \\
\hline 1 & Aguacate & Persea americana & $x$ & & $x$ & $x$ \\
\hline 2 & Almendro & Terminalia cattapa & & & $x$ & $x$ \\
\hline 3 & Almirajó & Patinoa almirajó & & & $x$ & \\
\hline 4 & Anón & Annona squamosa & & & $x$ & \\
\hline 5 & Arbol del pan & Artocarpus altilis & & & $x$ & $x$ \\
\hline 6 & Bacao & Theobroma bicolor & & & $x$ & \\
\hline 9 & Bija & Bixa orellana & & & $x$ & $x$ \\
\hline 10 & Caimito & Pouteria caimito & & & $x$ & \\
\hline 11 & Chirimoya & Annona cherimola & & & $x$ & \\
\hline 12 & Chontaduro & Bactris gasipaes & $x$ & $x$ & $x$ & $x$ \\
\hline 13 & Coco & Cocos nucifera $L$. & $x$ & & $x$ & \\
\hline \multirow[t]{2}{*}{14} & Coronillo & Bellusia glossularoides & & & $x$ & \\
\hline & Guadua & Guadua angustifolia & & & & $x$ \\
\hline 16 & Guamos & Inga edulis & $x$ & $x$ & $x$ & $x$ \\
\hline 17 & Guanábana & Annona muricata & & & $x$ & \\
\hline 21 & Guayaba agria & Psidium araca & & & $x$ & \\
\hline 22 & Guayaba dulce & Psidium guajaba & & & $x$ & \\
\hline 23 & Marañón & Zisigium macensis & & & $x$ & \\
\hline 24 & Malvavisco & Malvaviscos spp & & & & $x$ \\
\hline 25 & Matarratón & Gliricidia sepium & $x$ & $x$ & & $\mathrm{x}$ \\
\hline 26 & Mil peso & Oenocarpus bataua & & & $x$ & \\
\hline 27 & Nacedero & Trichanthera gigantea & $x$ & & & $x$ \\
\hline 28 & Pacó & Gustavia nana & & & $x$ & \\
\hline 29 & Yarumo & Cecropia peltata & $x$ & & & $x$ \\
\hline 30 & Anón & Anona muricata & & & $x$ & \\
\hline
\end{tabular}

cuentra en $95 \%$ de los huertos caseros mixtos. Los frutos de Carica papaya son muy apetecidos a la hora de preparar jugos y para consumo directo, poseen además una alta demanda de comercialización. El fruto de Citrus lemon es uno de los que más utiliza el hombre porque además de que se requiere para la preparación de jugos y de otros tipos de alimentos, se emplea en la preparación de remedios caseros para tratar algunas afecciones como la gripa, parásitos, dolor de estómago, sin olvidar también que muchas personas lo usan con algunos fines mágico religiosos. Los valores de CIE para las especies de este hábito se muestran en la Tabla 5.

Estrato herbáceo. Eringyum foetidum y Ocimum $S p$, además de ser condimentos de alta calidad para la preparación de las comidas, conocidos en el ámbito regional nacional e internacional, son muy utilizadas de manera local con fines mágico-religiosos y medicinales. Petiveria alliacea a pesar de que no tiene demanda de comercialización ni un uso alimenticio, justifica su importancia porque está presente en huertos caseros mixtos, azoteas, otros utensilios y patios, frontales y posteriores, lo que se determina por las diversas propiedades medicinales, mágico-religiosas y ornamentales. La composición y nivel de importancia de uso de las especies de este hábito se muestran en la Tabla 6 .

En la Tabla 7 se muestran las principales especies de acuerdo con su CIE por hábito de crecimiento, cuyos valores se pueden comparar con los de otras especies consignadas en el Tabla 4. En la Figura 4 se puede visualizar el coeficiente de importancia de las especies más sobresalientes dentro de los sistemas de producción urbana de Quibdó.

Prácticas de manejo utilizadas en los sistemas produc- 
Bioetnia Volumen 7 № 1 (enero-junio), 2010

Tabla 2

Especies encontradas según hábito arbustivo

\begin{tabular}{|c|c|c|c|c|c|c|}
\hline \multirow{2}{*}{$\begin{array}{c}\text { Hábito ecológico } \\
\text { Arbustivo }\end{array}$} & \multirow[t]{2}{*}{ Nombre local } & \multirow[t]{2}{*}{ Nombre científico } & \multicolumn{4}{|c|}{ Nivel de utilización } \\
\hline & & & Medicinal & Mágico religiosa & Alimento & Otros \\
\hline 1 & Arazá & Eugenia stipitata & & & $x$ & \\
\hline 2 & Borojó & Borojoa patinoi & $x$ & $x$ & $x$ & $x$ \\
\hline 3 & Cacao & Theobroma cacao & & & $x$ & \\
\hline 4 & Caña & Saccharum officinarum & & & $x$ & \\
\hline 5 & Carambolo & Averrhoa carambola & & & $x$ & \\
\hline 6 & Guanábana & Annona muricata & & & $x$ & \\
\hline 7 & Limón & Citrus lemon & $x$ & $x$ & $x$ & $x$ \\
\hline 8 & Palma de Cristo & Cardyline terminalis & & $x$ & & $x$ \\
\hline 9 & Papaya & Carica papaya & & & $x$ & \\
\hline 10 & Plátano & Musa spp & & & $x$ & \\
\hline 11 & Sauco & Solanum incoptum & $x$ & & & \\
\hline 12 & Totumo & Crescentia cujete & $x$ & & & $x$ \\
\hline 13 & Noni & Morinda citrifolia & $x$ & & & $\mathrm{x}$ \\
\hline
\end{tabular}

Tabla 3

Especies encontradas según hábito arbustivo

\begin{tabular}{|c|c|c|c|c|c|c|}
\hline \multirow{2}{*}{$\begin{array}{c}\text { Hábito ecológico } \\
\text { Herbácea }\end{array}$} & \multirow[t]{2}{*}{ Nombre local } & \multirow[t]{2}{*}{ Nombre científico } & \multicolumn{4}{|c|}{ Nivel de utilización } \\
\hline & & & Medicina & Mágico religiosa & Alimer & tros \\
\hline 1 & Achín & Xantosoma sagitifolium & & & $\mathrm{x}$ & \\
\hline 2 & Albahaca & Ocimum sp & $x$ & $x$ & $x$ & $\mathrm{x}$ \\
\hline 4 & Badea & Passiflorasp & & & $x$ & \\
\hline 5 & Caña agria & Costus villosissimus & $x$ & & $x$ & \\
\hline 6 & Cilantro cimarrón & Eringyum foetidum & $x$ & & $x$ & \\
\hline 7 & Lulo & Solanum sp & & & $x$ & \\
\hline 8 & Piña & Ananas comosus & & & $x$ & \\
\hline 9 & Santa María & Piper peltatum & $x$ & $x$ & & \\
\hline 10 & Amansa justicia & Justicia pectoralis & & $\mathrm{x}$ & & $\mathrm{x}$ \\
\hline 11 & Poleo & Mentha pulegium & & & $x$ & \\
\hline 12 & Ortiga & Urtica urens L. & & & & $x$ \\
\hline 13 & Anamú & Petiveria alliacea L & & $x$ & & $\mathrm{x}$ \\
\hline 14 & Paico & Chenopodium ambrosioides & $x$ & & & \\
\hline 15 & Menta & Mentha piperita & $x$ & & & \\
\hline 16 & Pipilongo & Piper sp & $x$ & & & $\mathrm{x}$ \\
\hline 17 & Jengibre & Zingiber officinale & $x$ & & $x$ & \\
\hline 18 & Limoncillo & Cymbopogon citratus & $x$ & & $x$ & $x$ \\
\hline 19 & Platanillo & Heliconia spp & & & & $x$ \\
\hline 20 & Tomate & Lycupersicon sculentum & & & $x$ & \\
\hline 21 & Cebolla de rama & Allium fistulosum & & & $\mathrm{x}$ & \\
\hline
\end{tabular}


Sistemas productivos urbanos. T Cuesta-Borja, DH Mosquera

Tabla 4

Composición florística y Coeficiente de Importancia de las Especies de hábito arbóreo (CIE)

\begin{tabular}{lllll}
\hline $\begin{array}{l}\text { Hábito ecológico } \\
\text { Arbóreo }\end{array}$ & Nombre local & Nombre científico & Familia & CIE \\
\hline & & & & \\
\hline & Aguacate & Persea americana & Lauraceae & 1,7 \\
2 & Almendro & Terminalia cattapa & Combretaceae & 1,3 \\
3 & Almirajó & Patinoa almirajó & Bombacaceae & 1,2 \\
4 & Anón & Annona squamosa & Anonaceae & 1,0 \\
5 & Arbol del pan & Artocarpus altilis & Moraceae & 1,5 \\
6 & Bacao & Theobroma bicolor & Sterculiaceae & 1,5 \\
7 & Bija & Bixa orellana & Bixaceae & 1,7 \\
8 & Caimito & Pouteria caimito & Sapotaceae & 1,2 \\
9 & Chirimoya & Annona cherimola & Annonaceae & 1,3 \\
10 & Chontaduro & Bactris gasipaes & Arecaceae & 3,0 \\
11 & Coco & Cocos nucifera L. & Arecaceae & 1,3 \\
12 & Coronillo & Bellusiaglossularoides & Melastomataceae & 0,8 \\
13 & Guadua & Guadua angustifolia & Poaceae & 1,0 \\
14 & Guamos & Inga edulis & Mimosaceae & 2,8 \\
15 & Guayaba agria & psidium araca & Mirtaceae & 1,3 \\
16 & Guayaba dulce & psidium guajaba & Mirtaceae & 1,5 \\
17 & Marañón & Zisigium malacensis & Mirtaceae & 2,0 \\
18 & Malvavisco & Malvaviscos spp & Malvaceae & 0,8 \\
19 & Matarratón & Gliricidia sepium & Fabaceae & 1,2 \\
20 & Mil peso & Oenocarpus bataua & Arecaceae & 1,0 \\
21 & Nacedero & Trichanthera gigantea & Verbenaceaea & 1,7 \\
22 & Pacó & Gustavia nana & Lecythidaceae & 1,3 \\
23 & Yarumo & Cecropia peltata & Cecropiaceae & 0,8 \\
\hline
\end{tabular}

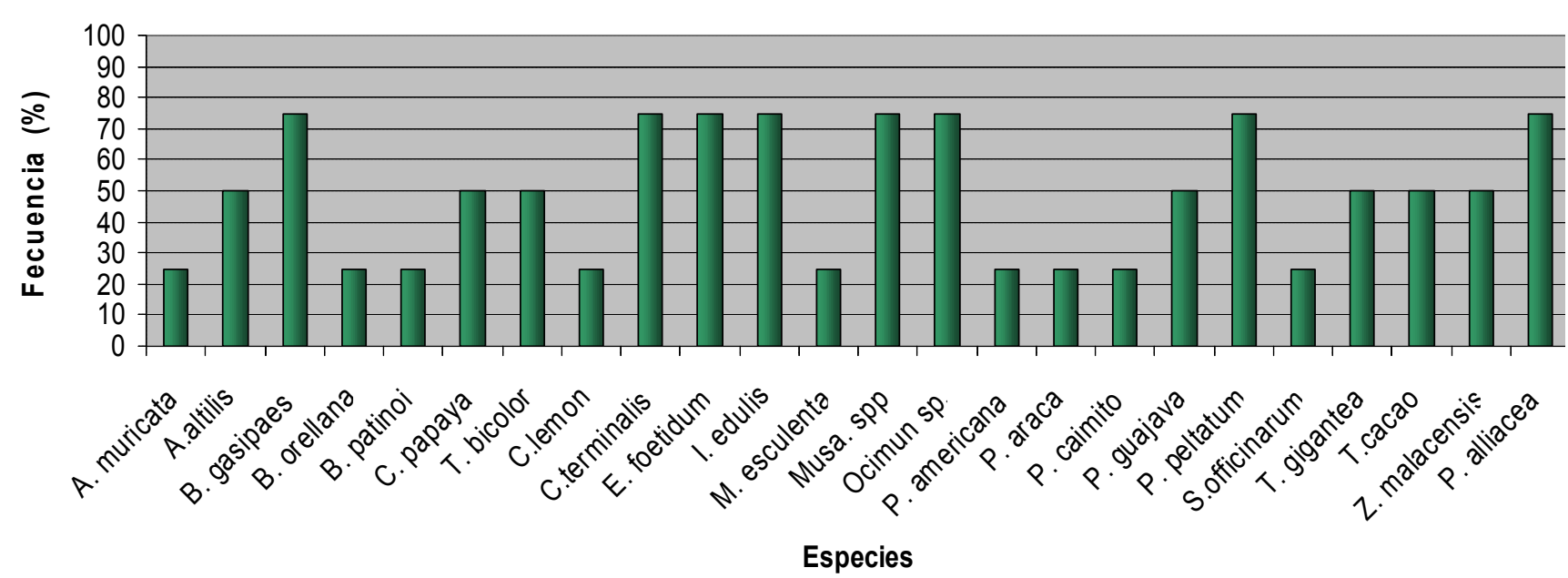

Figura 3. Diagrama de frecuencia de las especies. 
Bioetnia Volumen 7 № 1 (enero-junio), 2010

Tabla 5

Composición florística y Coeficiente de Importancia de las Especies (CIE) para el hábito arbustivo

\begin{tabular}{cllll}
\hline $\begin{array}{c}\text { Hábito ecológico } \\
\text { Arbustivo }\end{array}$ & Nombre local & Nombre científico & Familia & CIE \\
\hline 1 & Arazá & Eugenia stipitata & Mirtaceae & 0,8 \\
2 & Borojó & Borojoa patinoi & Rubiaceae & 2,2 \\
3 & Cacao & Theobroma cacao & Sterculiaceae & 1,5 \\
4 & Caña & Saccharum officinarum & Poaceae & 1,2 \\
5 & Carambolo & Averrhoa carambola & Oxalidaceae & 1,2 \\
6 & Guanábana & Annona muricata & Anonaceae & 1,7 \\
7 & Limón & Citrus lemon & Rutacea & 2,2 \\
8 & Palma de Cristo & Cardyline terminalis & Liliaceae & 2,0 \\
9 & Papaya & Carica papaya & Caricaceae & 2,7 \\
10 & Plátano & Musa spp & Musaceae & 3,0 \\
11 & Sauco & Solanum incoptum L & Solanaceae & 2,0 \\
12 & Totumo & crescentia cujete & Bignoniaceae & 1,8 \\
13 & Noni & Morinda citrifolia & Rubiaceae & 1,5 \\
14 & Pipilongo & Piper sp & Piperaceae & 1,3 \\
\hline
\end{tabular}

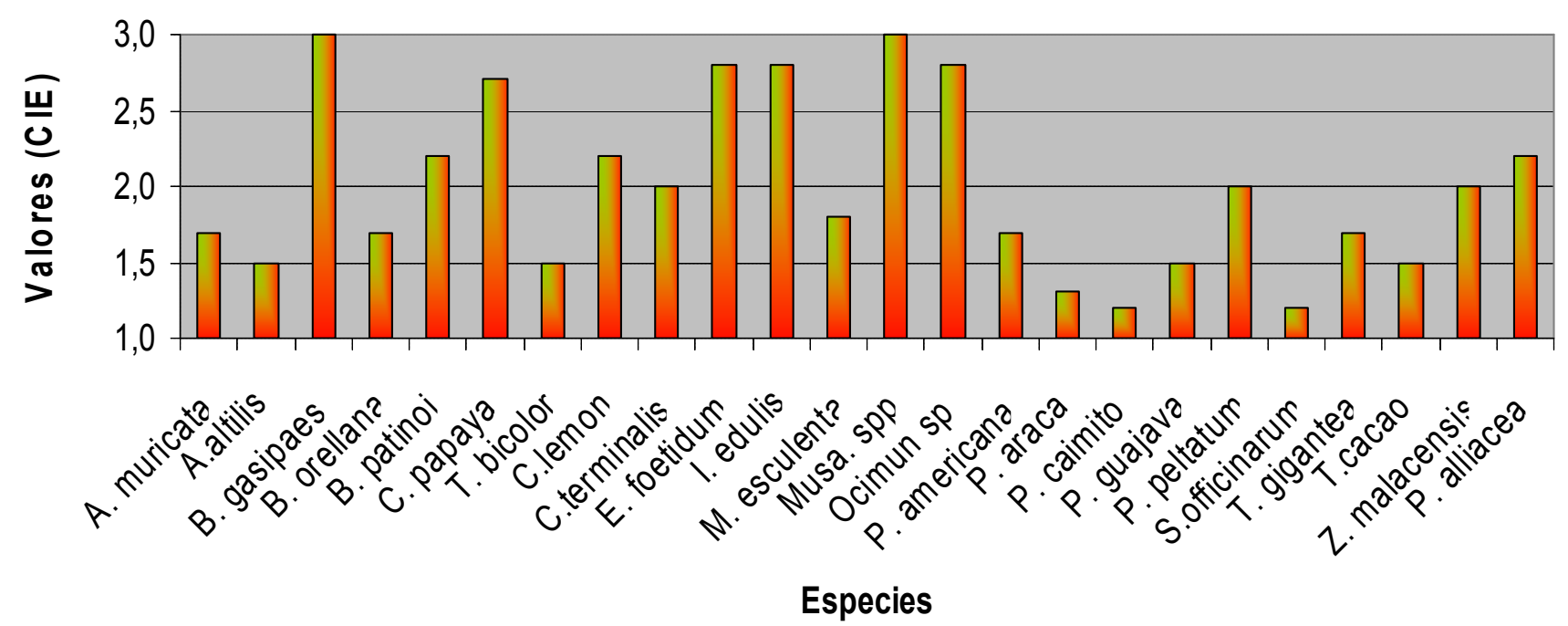

Figura 4. Coeficiente de importancia de algunas de las especies encontradas.

tivos urbanos de la ciudad de Quibdó. Entre las principales prácticas de manejo encontradas en los sistemas productivos urbanos se encuentran el abonado, las podas sanitarias y estéticas, las resiembras, rocerías y el control de plagas.

El abonado. El abonado o fertilización de los huertos caseros se realiza por lo general de manera orgánica, proveniente de residuos de cocina, de residuos de cosecha, hojarasca o cualquier material biodegradable de origen natural, que se aplica de forma directa en la base de árboles y arbustos sobre todo en guama, plátano, guanábana, etc. El sustrato tradicionalmente usado para la siembra de plantas en azoteas es la tierra de hormiga (desechos de Atta sp.), aunque de manera reciente se ha empezado a utilizar la gallinaza, producto de las actuales explotaciones avícolas que se encuentran en los alrededores de la ciudad.

La poda sanitaria y estética. Se realiza básicamentee para 
Tabla 6

\section{Composición florística y Coeficiente de Importancia de las Especies (CIE) para el hábito herbáceo}

\begin{tabular}{cllll}
\hline $\begin{array}{c}\text { Hábito ecológico } \\
\text { Herbáceo }\end{array}$ & Nombre local & Nombre científico & Familia & CIE \\
\hline 1 & Achín & Xantosoma sagitifolium & Araceace & 1,3 \\
2 & Albahaca & Ocimum sp & Labiatae & 2,8 \\
3 & Badea & reasora sp & Passifloraceae & 1,5 \\
4 & Caña agria & Costus villosissimus & & 1,3 \\
5 & Cilantro cimarrón & Eringyum foetidum & Apiaceae & 2,8 \\
6 & Lulo & Solanum sp & Solanaceae & 1,5 \\
7 & Piña & Ananas comosus & Bromeliaceae & 1,7 \\
8 & Santa María & Piper peltatum & Piperaceae & 2,0 \\
9 & Amansa justicia & Justicia pectoralis & Acanthaceae & 1,3 \\
10 & Poleo & Mentha pulegium & Lamiaceae & 1,5 \\
11 & Ortiga & Urtica reas L. & Urticaceae & 1,7 \\
12 & Anamú & Petiveria alliacea L & Alliaceae & 2,2 \\
13 & Paico & Chenopodium ambrosioides & Chenopodiaceae & 1,5 \\
14 & Menta & Mentha piperita & Lamiaceae & 1,5 \\
15 & Jengibre & Zingiber officinale & Zingiberaceae & 2,0 \\
16 & Limoncillo & Cymbopogon citratus & Poaceae & 1,3 \\
17 & Platanillo & Heliconia spp. & Heliconeaceae & 0,8 \\
18 & Tomate & Lycupersicon sculentum & Solanaceae & 1,3 \\
19 & Cebolla de rama & Allium fistulosum & Alliaceae & 1,3 \\
20 & Yuca & Mahihot sculenta & Euphorbiaceae & 1,8 \\
\hline
\end{tabular}

Tabla 7

Especies más importantes entre los hábitos ecológicos estudiados

\begin{tabular}{cllll}
\hline Hábito ecológico & Especie & Familia & Nombre local & CIE \\
\hline \multirow{2}{*}{ Arbóreo } & Bactris gasipaes & Arecaceae & Chontaduro & 3,0 \\
& Inga edulis & Mimosaceae & Guamo & 2,8 \\
& Zisigyum malacensis & Mirtaceeae & Marañón & 2,0 \\
\multirow{2}{*}{ Arbustivo } & Carica papaya & Rubiaceae & Borojó & 2,7 \\
& Citrus lemon & Rutacea & Limón & 2.2 \\
& Musa spp & Musaceae & Plátano & 2,7 \\
Herbáceo & Eringyum foetidum & Apiaceae & Cilantro & 2.8 \\
& Ocimum sp & Labiatae & Albahaca & 2.8 \\
& Petiveria alliacea & Alliaceae & Anamú & 2.2 \\
\hline
\end{tabular}




\section{Bioetnia Volumen 7 № 1 (enero-junio), 2010}

eliminar estructuras viejas o enfermas de plantas ornamentales y alimenticias, entre las que están: plátano, plantas de azotea, plantas ornamentales, entre otras. La poda estética es el recorte de los tallos y ramas con fines decorativos (para darles determinadas formas). Se hace por lo general en plantas ornamentales (arbustos) ubicadas en el frente de las casas.

Resiembra. Se realiza para reemplazar plantas que han muerto por motivos sanitarios o mecánicos; su aplicación se realiza independiente de la especie vegetal cultivada, es decir en arbóreas, arbustivas o herbáceas.

Rocerías (control de malezas o arvenses). Con el objetivo de eliminar la competencia intraespecífica e interespecífica de las especies cultivadas, mejorar el aspecto del sistema o facilitar la movilidad dentro de éste, se realiza el control de la vegetación «indeseable» cada 2-3 meses. Esta práctica se realiza con machete.

Control de plagas. Son numerosas las plagas que se pueden encontrar en los sistemas productivos de la ciudad de Quibdó, las que en su mayoría no representan una amenaza económica actual, por lo que en general su control se realiza por medios mecánicos (remoción, eliminación manual). No obstante, la plaga más sentida por los agricultores urbanos, es la hormiga arriera Atta sp y acromirmex, que forrajea (desfolia, corta) las hojas y ramas de todo tipo de plantas (arbóreas, arbustivas, herbáceas). En muchos casos, para su control, los agricultores recurren a insecticidas comerciales cono es el caso del Arrierafín, Arriero y Attakill.

\section{CONCLUSIONES}

Los sistemas productivos urbanos en la ciudad de Quibdó constituyen una actividad tradicional, que está orientada fundamentalmente a suplir necesidades alimentarias y medicinales; estos sistemas de producción están distribuidos en tres estratos, el arbóreo, el arbustivo y el herbáceo. Sin embargo, estos sistemas no se manejan con criterios técnicos, razón por la que los rendimientos son bajos frente a los estándares de producción de los cultivos en Colombia.

\section{LITERATURA CITADA}

FAO. 1999. La agricultura urbana y periurbana. 150 periodos de sesiones. Comité de Agricultura. Roma: Food Agriculture Organization. En línea. URL disponible en: www.fao.org.co

Gama, J. R. V. 1995. Comunidades de Ribeirinhos no NE paraense. Nota Técnica $\mathrm{N}^{\circ}$ 2. Belém: Projeto VÁRZEA; $5 \mathrm{pp}$.

Mougeot, L. 1993. Autosuficiencia alimentaria urbana: significación y retos. CIID Informa. 21 (3): 2-5.

Rangel, J. 2004. Colombia diversidad biótica IV. El Chocó biogeográfico/ costa Pacifica. Bogotá, DC: Universidad Nacional de Colombia; p. 599615.

SIG-UTCH. 2006. Mapa de la localización geográfica del municipio de Quibdó Quibdó: Universidad Tecnológica del Chocó. 